\section{Gürtelrose ist unter Statintherapie häufiger}

\author{
Bereits aus früheren Studien geht hervor, dass bei Patienten mit einer \\ Statintherapie die Wahrscheinlichkeit für die Entwicklung eines Herpes \\ zoster leicht erhöht ist. Unklar war bisher, ob dies dosisabhängig ist. Dieser \\ Zusammenhang wurde nun überprüft.
}

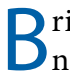
ritische Epidemiologen haben in einer Fall-Kontroll-Studie auf der Basis des U.K. Clinical Practice Research Datalink den Zusammenhang von Gürtelrose-Risiko unter Statintherapie untersucht. In die Datenbank flossen Patienteninformationen von bisher $9 \%$ der britischen Bevölkerung ein. Für die aktuelle Analyse standen die Befunde von knapp 145.000 Zoster-Patienten und fast 550.000 Teilnehmern der gematchten Kontrollgruppe zur Verfügung. Sie waren mindestens 18 Jahre alt. Das Follow-up reichte von Januar 2000 bis Dezember 2011.

In der Multivariatanalyse berücksichtigten die Wissenschaftler mehrere potenzielle Risikofaktoren für die Entwicklung einer Gürtelrose. Dazu gehörten neben Übergewicht, Rauchen und Alkoholkonsum auch HIV-Infektion, Leukämie und Myelom sowie Stammzelltrans- plantation, Depression, COPD und Diabetes. Dem Ergebnis der Regressionsanalyse zufolge war die Wahrscheinlichkeit, während einer Statintherapie an einer Gürtelrose zu erkranken, um 13\% erhöht (Odds Ratio [OR]: 1,13; 95\%-Konfidenzintervall 1,11-1,15). Ähnliche Ergebnisse waren bereits in früheren Studien errechnet worden.

Je länger das Ende der Statintherapie zurücklag, umso geringer war schließlich das Zosterrisiko. Waren seitdem bis zu zwölf Monate vergangen, lag die OR noch bei 1,08 (95\%-KI 1,04-1,13), nach mehr als drei Jahren nur noch bei 1,06 (95\%-KI 0,99-1,14). Dies sei ein Hinweis auf einen kausalen Zusammenhang zwischen Statintherapie und Gürtelrose, so die Autoren. Der Mechanismus sei allerdings noch unklar. Es wird unter anderem vermutet, dass über eine Abnahme der GTPase-Aktivität die Aktivierung der T-Zellen und deren Proliferation geschwächt sind.

Die Wissenschaftler weisen darauf hin, dass es zwar auch eine Assoziation zwischen ACE-Hemmer-Therapie und Zosterentstehung gibt. Allerdings falle die Erhöhung des Erkrankungsrisikos im Vergleich zu Patienten, die nie damit behandelt wurden, kaum ins Gewicht (OR: 1,03; 95\%-KI 1,01-1,05). Die Einnahme von ACE-Hemmern, die oft gemeinsam mit Statinen verschrieben werden, sei deshalb nicht der Grund für das erhöhte Zosterrisiko unter den Statinen, so die Forscher.

Fazit: Die Behandlung mit Statinen ist mit einem erhöhten Risiko assoziiert, an einem Herpes zoster zu erkranken, und zwar dosisabhängig: Je länger das Ende einer Statintherapie zurückliegt, umso geringer wird die Wahrscheinlichkeit einer Gürtelrose.

Peter Leiner

Matthews A et al. Statin use and the risk of herpes zoster: a nested case-control study using primary care data from the U.K. Clinical Research Practice Datalink. Br J Dermatol 2016; 175: 1183-94

\title{
Wissen Sie, was ein Kerion ist?
}

Ein 13-jähriges Mädchen hatte eine langsam wachsende, schmerzhafte Schwellung der Kopfhaut des Hinterkopfs, die sich im Laufe von drei Wochen entwickelt hatte. Wegen des Verdachts auf eine bakterielle Follikulitis hatte sie mehrere Behandlungen mit oralen Antibiotika hinter sich, ohne dass sich der Befund gebessert hätte. Bei der ersten Vorstellung zeigten sich eine gerötete, leicht schmerzhafte Schwellung am Hinterkopf mit pustulösen Veränderungen und einem serös-blutigen Exsudat sowie eine vollständige Alopezie (Abb. a). Ein Schabepräparat der betroffenen Stelle und eine Präparation in 10\%iger Kaliumhydroxidlauge brachte Pilzhyphen zum Vorschein und bestätigte die Diagnose eines Kerions.

Ein Kerion ist eine entzündliche Pilzinfektion der Kopfhaut, die man üblicherweise bei Kindern sieht. Da sie exsudativ und schmerzhaft ist und auch mit einer regionären Lymphadenopathie einhergehen kann, wird sie oft mit einer bakteriellen Follikulitis verwechselt. Die häufigsten Erreger sind Trichophyton verrucosum und Trichophyton mentagrophytes. Meistens verwendet man zur Therapie orales Griseofulvin, obgleich auch andere Antimykotika effektiv sind. Eine Verzögerung von Diagnose und Behandlung führt zu einer narbigen Alopezie. Die Patientin wurde über sechs Wochen hinweg mit Griseofulvin behandelt, worauf sich der Befund deutlich besserte (Abb. b). Die narbige Alopezie wird aber zumindest teilweise persistieren.

Prof. Hermann FüeßI

Uprety S et al. Kerion — a boggy lump. N Engl J Med 2016; 375: 980
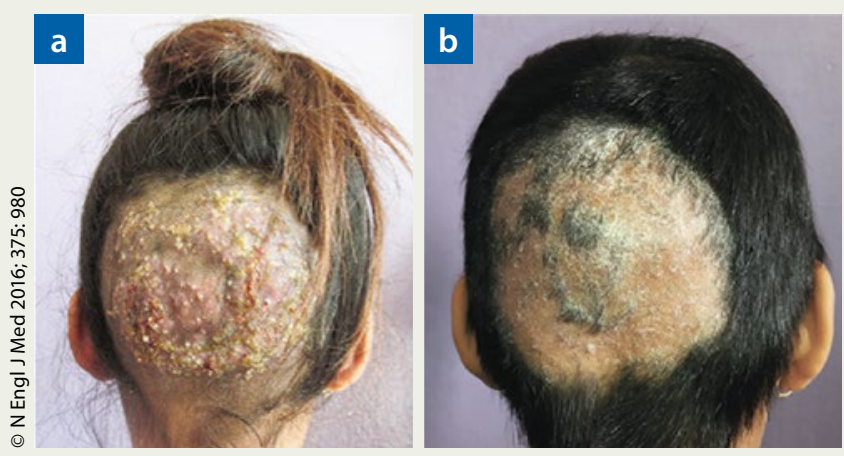

a: Schwellung am Hinterkopf mit Pusteln, serös-blutigem Exsudat und Alopezie; b: Zustand nach sechs Wochen 